\title{
Advanced Stages of Medication-Related Osteonecrosis of the Jaw: From Diagnosis to Surgical Treatment and Rehabilitation with Removable Prosthesis ${ }^{\dagger}$
}

\author{
Angela Tempesta *, Luisa Limongelli, Saverio Capodiferro, Massimo Corsalini and Gianfranco Favia \\ Department of Interdisciplinary Medicine, University of Bari, 70124 Bari, Italy; \\ luisanna.limongelli@gmail.com (L.L.); capodiferro.saverio@gmail.com (S.C.); massimo.corsalini@uniba.it \\ (M.C.); gianfranco.favia@uniba.it (G.F.) \\ * Correspondence: angelatempesta1989@gmail.com; Tel.: +39-0805218784 \\ + Presented at the XV National and III International Congress of the Italian Society of Oral Pathology and \\ Medicine (SIPMO), Bari, Italy, 17-19 October 2019.
}

Published: 12 December 2019

Medication-related osteonecrosis of the Jaw (MRONJ) is an uncommon but potentially serious side effect of treatment with antiresorptive or antiangiogenic drugs among oncologic and osteoporotic patients. Clinical examination and radiological exams are mandatory for diagnosis and staging; nevertheless, even after II level exams, as Computed Tomography, MRONJ understaging is frequent [1]. Moreover, the association with pus discharge, chronic sinusitis and neurologic pathology is frequent, especially for Stage II and III lesions. To date, the treatment is still controversial, but many studies in the current literature state that only surgical removal of necrotic bone guarantees complete healing, especially for high stage lesions [1]. This surgical approach produces severe bone loss, with difficult aesthetic and functional prosthetic rehabilitation, because bone regeneration with autologous or heterologous graft cannot be performed among these patients [2]. The aim of the current study was to describe diagnostic and therapeutic protocol for patients affected by MRONJ, and subsequent rehabilitation with removable prosthesis (RP).

For the current study, authors selected 41 patients from the database of MRONJ of the Complex Operating Unit of Odontostomatology comprehending 301 patients with 389 treated lesions. The inclusion criteria were:

1. Diagnosis of Stage III MRONJ according with AAOMS criteria

2. Surgical treatment of MRONJ with complete healing after not less than 6-months follow-up

3. Total edentulism of upper and/or lower jaw after treatment

All lesions were diagnosed and treated according to our protocol. Patients' anamnestic data were collected; Rx OPT and multislice spiral CT with 3D reconstruction were performed for the staging. Patients underwent marginal bone resection including at least $1 \mathrm{~cm}$ of vascularized bone tissue. The depth of resection was pinpointed by the bleeding evaluation of bone tissues. Surgery was complemented by using piezosurgical device for osteoplasty and with the application of a medical device made of hyaluronic acid and amino acids. Subsequently, after complete lesions healing without recurrence, the selected patients underwent prosthetic evaluation and RP were inserted. All dentures underwent relining with soft materials to reduce risk of trauma on gingival tissue, and patients underwent periodic follow-up (once-a-month for the first year, then 3-times a year).

Overall, after a follow-up time of not less than 3 years, all patient showed complete healing of MRONJ without signs and symptoms of recurrence. Removable prosthesis allowed a good functional and aesthetic rehabilitation, with patients' satisfaction. Periodic relining with soft materials were 
performed (generally every 3 months). Decubitus ulcers of oral mucosa were evaluated, especially after the first insertion of prosthesis, but they always healed after dentures adjustments and relining. In these cases, application of a gel compound with hyaluronic acid and amino acids was prescribed to accelerate wound healing thus reducing the risk of MRONJ occurrence. In no one case MRONJ onset was evaluated.

Advanced Stages of MRONJ always require surgical treatment producing severe bone loss with aesthetic and functional deficit. In these cases, RP could be valid solution for patients. Nevertheless, considering that ill-fitting RP could produce MRONJ, periodic relining with soft materials and continuous adjustment are mandatory to avoid this complication.

Conflicts of Interest: The authors declare no conflict of interest.

\section{References}

1. Favia, G.; Tempesta, A.; Limongelli, L.; Crincoli, V.; Maiorano, E. Medication-related osteonecrosis of the jaw: Surgical or non-surgical treatment? Oral Dis. 2018, 24, 238-242.

2. Zirk, M.; Kreppel, M.; Buller, J.; Pristup, J.; Peters, F.; Dreiseidler, T.; Zinser, M.; Zöller, J.E. The impact of surgical intervention and antibiotics on MRONJ stage II and III-Retrospective study. J. Craniomaxillofac. Surg. 2017, 45, 1183-1189.

(C) 2019 by the authors. Licensee MDPI, Basel, Switzerland. This article is an open access article distributed under the terms and conditions of the Creative Commons Attribution (CC BY) license (http://creativecommons.org/licenses/by/4.0/). 